\title{
Sutureless pneumostasis using bioabsorbable mesh and glue during major lung resection for cancer: Who are the best candidates?
}

\author{
Kazuhiro Ueda, MD, ${ }^{\mathrm{a}}$ Toshiki Tanaka, MD, ${ }^{\mathrm{a}}$ Tao-Sheng Li, MD, ${ }^{\mathrm{a}}$ Nobuyuki Tanaka, MD, ${ }^{\mathrm{b}}$ and \\ Kimikazu Hamano, MD ${ }^{\mathrm{a}}$
}

\begin{abstract}
Objective: Preventing air leaks after major lung resection for cancer is mandatory for successful fast-track surgical intervention. We reported our preliminary results with performance of pneumostasis by combining polyglycolic acid mesh and fibrin glue; however, the advantages of this combination over the conventional method have not been clarified.
\end{abstract}

\begin{abstract}
Methods: We controlled air leaks detected during an intraoperative water-seal test by using sutures and fibrin glue before April 2006 and by combining polyglycolic acid mesh and fibrin glue without sutures thereafter. We removed the chest tube the day after the air leaks stopped. For bias reduction in comparison with the 2 historical cohorts, we used the nearest available matching method with the estimated propensity score.
\end{abstract}

Results: The durations of chest tube drainage and postoperative hospital stay were significantly shorter in the mesh-and-glue group $(n=61)$ than in the glue-alone group $(n=61)$. The incidence of postoperative pulmonary complications was lower in the mesh-and-glue group than in the glue-alone group $(0 \%$ vs $7 \%, P=.042)$. According to a stratification analysis, the benefit of combining mesh and glue to reduce the duration of chest tube drainage was limited in patients undergoing upper lobe resection and in patients with severe emphysema undergoing other types of resection.

Conclusion: Combining bioabsorbable mesh and glue for pneumostasis can reduce the duration of chest tube drainage, postoperative hospital stay, and pulmonary complications after major lung resection for cancer. Patients undergoing upper lobe resection and those with severe emphysema might be the best candidates for this technique. (J Thorac Cardiovasc Surg 2010;139:600-5)

The prevention of air leaks after major lung resection for cancer is essential to a successful early postoperative outcome. Although many investigators have attempted to close air leaks with various surgical sealants, there is still not enough evidence to support the use of sealant. ${ }^{1}$ Recently, we reported the preliminarily results of a procedure used to close air leaks by combining polyglycolic acid (PGA) mesh and fibrin glue. ${ }^{2}$ However, the advantages of this combination over standard methods remain unclear. In the present study we compared the postoperative outcomes of patients who underwent pneumostasis using suturing and glue with those of patients who underwent pneumostasis using PGA mesh and glue but without suturing. We also attempted to identify which patients would benefit most from pneumostasis with the combination of PGA mesh and glue.

From the Department of Surgery and Clinical Science, Division of Chest Surgery, ${ }^{a}$ and the Department of Radiopathology and Science, Division of Radiology, ${ }^{\mathrm{b}}$ Yamaguchi University Graduate School of Medicine, Yamaguchi, Japan.

Disclosures: None

Received for publication Feb 11, 2008; revisions received May 24, 2009; accepted for publication June 21, 2009; available ahead of print Aug 5, 2009.

Address for reprints: Kazuhiro Ueda, MD, Department of Surgery and Clinical Science, Division of Chest Surgery, Yamaguchi University Graduate School of Medicine, 1-1-1 Minami-Kogushi, Ube, Yamaguchi 755-8505, Japan (E-mail: kaueda@ c-able.ne.jp).

$0022-5223 / \$ 36.00$

Copyright $(9) 2010$ by The American Association for Thoracic Surgery

doi:10.1016/j.jtcvs.2009.06.021

\section{MATERIALS AND METHODS}

\section{Patients}

This retrospective study was approved by the Institutional Review Board of the Yamaguchi University School of Medicine. Between April 2003 and December 2007, 145 patients who underwent major lung resection for diagnosed or suspected lung malignancy at our institution were included in this study. Patients who had undergone prior thoracotomy $(n=4)$ and those who underwent bronchoplasty $(n=3)$ were not included.

Operability was based on the existing guidelines for pulmonary resection. ${ }^{3}$ The criteria for resection included a $\mathrm{PaCO}_{2}$ of $50 \mathrm{~mm} \mathrm{Hg}$, a mean pulmonary arterial pressure of less than $30 \mathrm{~mm} \mathrm{Hg}$, and a calculated, predicted, postoperative forced expiratory volume in 1 second $\left(\mathrm{FEV}_{1}\right)$ of greater than $500 \mathrm{~mL}$. Patient data obtained preoperatively included age, sex, smoking habits, site of resection, and spirometric variables (ie, vital capacity and percent predicted $\mathrm{FEV}_{1}$ ). Upper lobe resection was defined as resection of at least the right upper lobe or the left upper division segment, and lower lobe resection was defined as resection of other parts of the lung. The patients' characteristics are summarized in Table 1.

Two materials were used for the pneumostasis: PGA mesh (Neoveil; Gunze, Osaka, Japan), a homopolymer nonwoven fabric with a molecular mass of 100,000, and fibrin glue (Bolheal; Chemo-Sero Therapeutic Institute, Kumamoto, Japan). The fibrin glue we used is composed of solution A and solution B. Solution A contains $80 \mathrm{mg} / \mathrm{mL}$ human fibrinogen, $75 \mathrm{U} / \mathrm{mL}$ human plasma-derived coagulation Factor XIII, and $1000 \mathrm{KIE}$ bovine aprotinin. Solution B contains $250 \mathrm{IU} / \mathrm{mL}$ human thrombin and $5.9 \mathrm{mg} / \mathrm{mL}$ calcium chloride. Mixing these 2 solutions forms a fibrin clot within 5 seconds.

\section{Pneumostasis and Chest Tube Management}

During major lung resection, we used an endoscopic stapler (Ethicon, Tokyo, Japan) to divide incomplete fissures and to excise the bronchus. 


\author{
Abbreviations and Acronyms \\ $\mathrm{CT}=$ computed tomographic \\ $\mathrm{FEV}_{1}=$ forced expiratory volume in 1 second \\ $\mathrm{HU}=$ Hounsfield units \\ $\mathrm{OR}=$ odds ratio \\ PGA $=$ polyglycolic acid \\ POD $=$ postoperative day
}

Staple lines were never reinforced. After resection, a water-seal test was done to ensure pneumostasis. We did not perform pleural tenting to obliterate residual pleural space after upper lobectomy. Before April 2006, we managed air leaks detected during the intraoperative water-seal test by suturing and sealing with fibrin glue alone; thereafter, we managed air leaks with fibrin glue in combination with PGA mesh without suturing in the following way.

Solution A was spread over the dissected lung parenchyma or the stapling line by rubbing it on the surface so that the fibrinogens could penetrate the lung parenchyma. Solution B was then spread over the surface to create primary sealing. This separate application of solution A and solution B with the rubbing procedure has been verified in cardiovascular surgery and neurosurgery in experimental models. ${ }^{4,5}$ A piece of PGA mesh, about $3 \times 3 \mathrm{~cm}$, soaked in solution A was placed over the sealed lung parenchyma and adhered by dropping solution B onto it. This process was repeated until all of the dissected area was adhered to the mesh. All these procedures were done with the residual lungs deflated.

A $20 \mathrm{~F}$ chest tube was placed in the hemithorax, and the wounds were closed. Postoperatively, chest tubes were attached to a continuous suction unit (MERA Sucuum; Mera Co Ltd, Tokyo, Japan) at $-10 \mathrm{~cm} \mathrm{H}_{2} \mathrm{O}$, which can suck at any pressure between 0 and $-50 \mathrm{~cm} \mathrm{H}_{2} \mathrm{O}$ as desired, and we checked for any sign of air leak by observing the suction device. If an air leak was detected, the chest tube suction was turned down in increments toward zero (water seal). Chest tubes were removed the day after the air leak disappeared, regardless of pleural drainage. This management plan was verified in a previous study. ${ }^{6}$

\section{Computed Tomographic Scanning and Emphysema Index}

Because the grade of emphysema is the most critical determinant for predicting prolonged air leakage after pulmonary resection, we routinely evaluated the grade of emphysema by using chest computed tomographic (CT) analysis in the following way.

A CT scan was performed with a 4-detector-row CT scanner (Siemens Volume Zoom; Siemens-Asahi Medical Ltd, Tokyo, Japan), with the patient in the supine position. The 2-mm-thick, high-resolution CT images of the entire lung were obtained in a $512 \times 512$ matrix during deep inspiratory breath holding with 2-mm collimation and a scan time of 1.0 seconds at 120 to $130 \mathrm{kVp}$ and 220 to $230 \mathrm{~mA}$. Transaxial CT images were reconstructed with the lung algorithm.

To evaluate the distribution and extent of emphysematous lung areas in each patient, we created volume-rendering 3-dimensional images of lung volume and low-attenuation areas, as well as density-masked images, which highlighted the lung areas with attenuation values of less than -910 Hounsfield units (HU), representative of emphysematous areas, by using imaging software (M900 QUADRA; Zio Soft K.K., Osaka, Japan). Threshold limits of -600 to $-910 \mathrm{HU}$ were applied to segment the entire lung and to exclude soft tissues surrounding the lung and the large vessels, atelectasis, fibrosis, and emphysematous areas within the lung. These 3-dimensional lung volume images represented lung contours, including lobar fissures, vasculature, and volume. They were used to quantify the area of emphysema with the emphysema index; that is, the percentage of the number of voxels with
TABLE 1. Clinical characteristics of the patients in the glue-alone and mesh-and-glue groups paired by using the nearest available matching method on the estimated propensity score

\begin{tabular}{llccc}
\hline \multicolumn{1}{c}{ Variables } & & $\begin{array}{c}\text { G group } \\
(\mathbf{n = 6 1 )}\end{array}$ & $\begin{array}{c}\text { MG group } \\
(\mathbf{n = 6 1 )}\end{array}$ & $\boldsymbol{P}$ value \\
\hline Age (y) & & $68 \pm 9$ & $67 \pm 10$ & .723 \\
Sex & Male & $39(64 \%)$ & $37(61 \%)$ & .709 \\
& Female & $22(36 \%)$ & $24(39 \%)$ & \\
Smoking history & No & $22(36 \%)$ & $23(38 \%)$ & .851 \\
& Yes & $39(64 \%)$ & $38(62 \%)$ & \\
Side of resection & Right & $36(59 \%)$ & $38(62 \%)$ & .711 \\
& Left & $25(41 \%)$ & $23(38 \%)$ & \\
Site of resection & Upper & $37(61 \%)$ & $41(67 \%)$ & .451 \\
& Lower & $24(39 \%)$ & $20(33 \%)$ & \\
Resected segments & & $4.1 \pm 1.2$ & $3.9 \pm 0.9$ & .203 \\
FVC (\%) & & $102 \pm 15$ & $103 \pm 13$ & .867 \\
FEV 1 (\%) & & $74 \pm 13$ & $73 \pm 13$ & .739 \\
Emphysema index & $<1 \%$ & $18(29 \%)$ & $18(29 \%)$ & .784 \\
& $1 \%-10 \%$ & $28(46 \%)$ & $31(51 \%)$ & \\
Intraoperative air leak & No & $15(25 \%)$ & $12(20 \%)$ & \\
& Yes & $24(38 \%)$ & $24(38 \%)$ & 1.000 \\
Propensity score & & $0.52 \pm 0.2$ & $0.55 \pm 0.1$ & .160 \\
\hline
\end{tabular}

Results are expressed as means \pm standard deviations unless otherwise specified. $G$, Glue alone; $M G$, mesh and glue; $F V C$, forced vital capacity; $F E V_{l}$, forced expiratory volume in 1 second.

attenuation values of less than $-910 \mathrm{HU}$ to the total number of voxels in the entire lungs $(-600$ to $-1024 \mathrm{HU}))^{7,8}$

\section{Statistical Analysis}

To reduce the bias in the comparison of a nonrandomized control group, we paired the treated group with the control group using the nearest available matching method on the estimated propensity score. The unpaired Student's $t$ test was used to test relationships between discrete variables and continuous variables. The $\chi^{2}$ test was used to compare discrete variables. The proportion of patients with chest tubes on each postoperative day (POD) was calculated and drawn by using the Kaplan-Meier method. Differences between the groups were calculated with the log-rank test. Stepwise multivariate Cox regression analysis was used to identify factors that independently influence the duration of chest tube drainage. All statistical analyses were performed with STATA 9.1 (StataCorp, College Station, Tex).

\section{RESULTS}

We originally collected data for a consecutive series of 61 patients who underwent surgical intervention before April 2006 and a consecutive series of 84 patients who underwent surgical intervention thereafter. We paired the 61 former patients (glue-alone group [G group]) with the 61 latter patients (PGA mesh-and-glue group [MG group]) using the nearest available matching method on the estimated propensity score. Table 1 shows the characteristics of $\mathrm{G}$ group and MG group patients. The propensity score was $0.623 \pm$ 0.148 for the original MG group $(\mathrm{n}=84)$, which was significantly different from that for the $\mathrm{G}$ group $(P<.0001)$. As shown in Table 1, no significant difference between the 2 groups was observed in the categorical and sequential 
variables, including the propensity score, after matching. The water-seal test detected an air leak intraoperatively in $37(61 \%)$ patients from the MG group, all of whom underwent pneumostasis with PGA mesh and glue. Air leaks disappeared immediately after pneumostasis, allowing chest tube removal on POD 1 in $55(90 \%)$ of the $61 \mathrm{MG}$ group patients. The air leaks eventually disappeared on POD 1 or 2 in the other 6 patients. The duration of chest tube drainage was significantly shorter in the MG group than in the $G$ group (mean of $1.1 \pm 0.4$ days and median of 1 day vs mean of $3.0 \pm 2.8$ days and median of 2 days, respectively; $P<.001$; Figure 1). The postoperative hospital stay was also significantly shorter for the $\mathrm{MG}$ group than for the $\mathrm{G}$ group (mean of $8.7 \pm 4.6$ days and median of 8 days vs mean of 11 \pm 5.8 days and median of 10 days, respectively; $P=.007$ ).

The emphysema index (odds ratio [OR] of 4.719 for emphysema index $<1 \%, P<0.001$; OR of 2.287 for emphysema index $1 \%-10 \%, P=.021)$ and the site of resection (OR of 2.176 for lower lobe resection, $P=.008$ ) were independent predictors for chest tube removal among the preoperative factors in $\mathrm{G}$ group patients, whereas none of the preoperative factors were associated with chest tube removal in the MG group patients. To identify which patients would be most likely to benefit from pneumostasis with PGA mesh, the patients were further stratified into 6 subgroups according to the site of resection (upper/lower) and the emphysema index $(<1 \% / 1 \%-10 \% />10 \%) .{ }^{9,10}$ The duration of chest tube drainage was shorter in the MG group than in the G group in patients with any emphysema index who had undergone upper lobe resection and in those with an emphysema index of greater than $10 \%$ who had undergone lower lobe resection (Figure 2). The duration of chest tube drainage and the postoperative hospital stay of patients with an air leak detected intraoperatively $(1.1 \pm 0.4$ days and $9.6 \pm 5,5$ days, respectively) were similar to those of patients without any air leak detected intraoperatively $(1.2 \pm 0.5$ days $[P=.605]$ and $7.4 \pm 2.4$ days $[P=.077]$, respectively) in $\mathrm{MG}$ group patients.

After chest tube removal, 5 of 122 patients required reinsertion for pneumothorax or subcutaneous emphysema at some point between POD 3 and POD 12 (median, POD 7). PGA mesh had been applied at the initial operation in 3 of these 5 patients. The air leaks disappeared spontaneously within a few days after redrainage in 4 patients but did not resolve in 1 patient, who required reoperation. This patient had undergone extensive adhesiolysis for diffuse intrapleural adhesions followed by right lower lobectomy at the initial operation. Despite complete pneumostasis with PGA mesh during the initial operation, an air leak developed where PGA mesh had not been applied. No other air leaks occurred within 30 days of observation after removal of the chest tubes.

Postoperative cardiopulmonary complications, excluding prolonged air leak, developed in $6(10 \%)$ patients in the $\mathrm{G}$

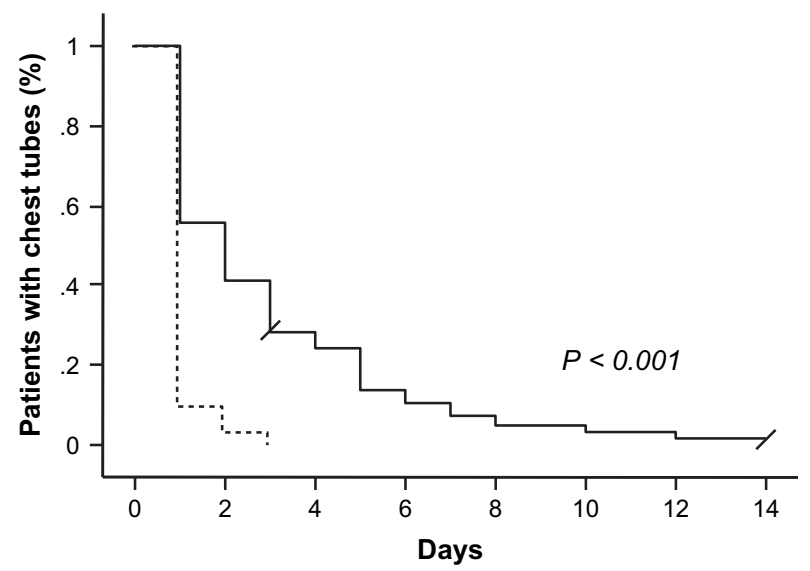

\begin{tabular}{lcccccccc} 
& \multicolumn{2}{l}{ No. at risk } \\
\cline { 1 - 2 } & 61 & 25 & 14 & 6 & 3 & 2 & 1 & 0
\end{tabular}

FIGURE 1. Percentage of patients with chest tubes on postoperative days 0 to 14 in the glue-alone $(\mathrm{G})$ group (solid line, $\mathrm{n}=61$ ) and the mesh-andglue (MG) group (dashed line, $\mathrm{n}=61$ ). Two patients in the $\mathrm{G}$ group required postoperative intervention for pulmonary complications before the air leak stopped (marked). The duration of chest tube drainage was significantly shorter in the MG group (mean of $1.1 \pm 0.4$ days and median of 1 day) than in the $\mathrm{G}$ group (mean of $3.0 \pm 2.8$ days and median of 2 days, $P<.001)$.

group and $2(3 \%)$ patients in the MG group $(P=.144$, Table 2). In particular, the incidence of pulmonary complications, including respiratory failure, lobar atelectasis, and pneumonia, was significantly lower in the MG group than in the $\mathrm{G}$ group $(0 \%$ vs $6.6 \%, P=.042)$.

\section{DISCUSSION}

The present retrospective review shows that the combination of PGA mesh and glue was superior to glue alone for reducing the duration of chest tube drainage, postoperative hospital stay, and postoperative cardiopulmonary complications. Furthermore, it allowed us to identify which patients would benefit from the combination of PGA mesh and glue. Our results might contribute to establishing the indication criteria for PGA mesh.

Postoperative air leaks occur frequently after closure of the chest wall, despite thorough water-seal testing and pneumostasis with glue alone. The possible mechanism of air leak recurrence could be that the residual lung becomes overinflated after closure of the chest wall, which stretches the dissected lung parenchyma, thereby reopening the alveolar fistula. The PGA mesh we used was a stretchable, soft, and thin $(0.15 \mathrm{~mm})$ fabric that was firmly adhered to the dissected lung surface with fibrin glue, attenuating excessive tension over the dissected area. The pliable and nonviscous properties of both solution A and solution B might also have played an important role in sealing alveolar air leaks, as well as in soaking the PGA mesh. 

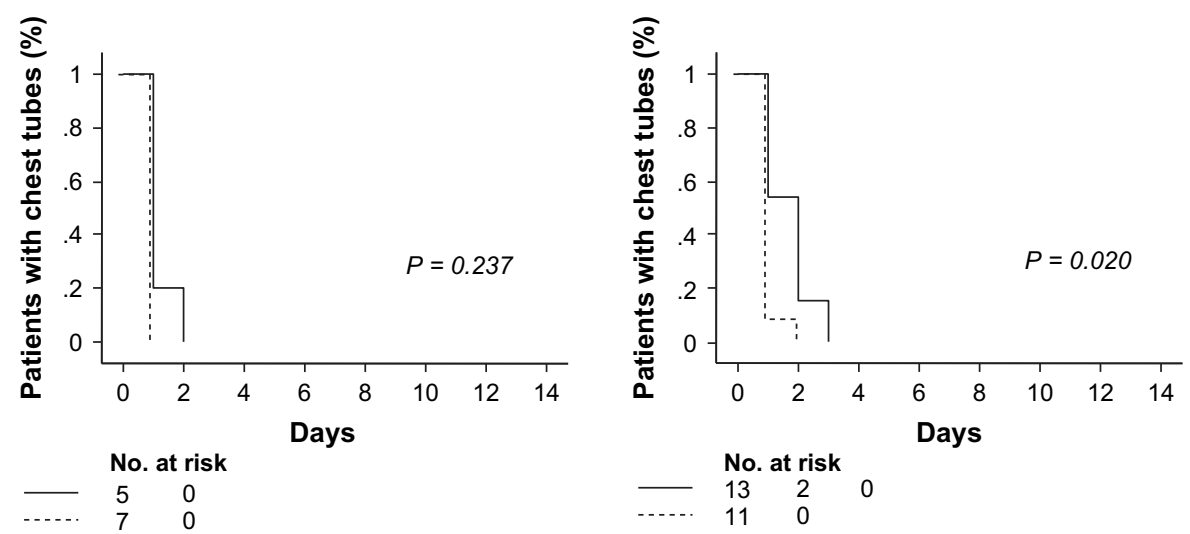

$$
\begin{array}{ll}
\text { No. at risk } \\
13 & 2 \\
11 & 0
\end{array}
$$

A
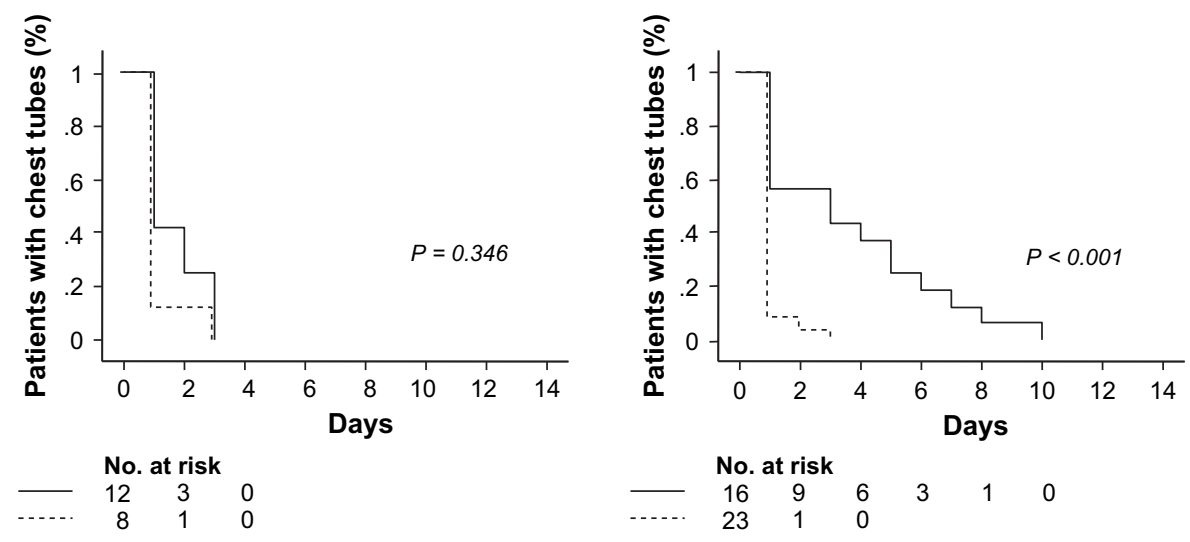

B

$\mathbf{E}$
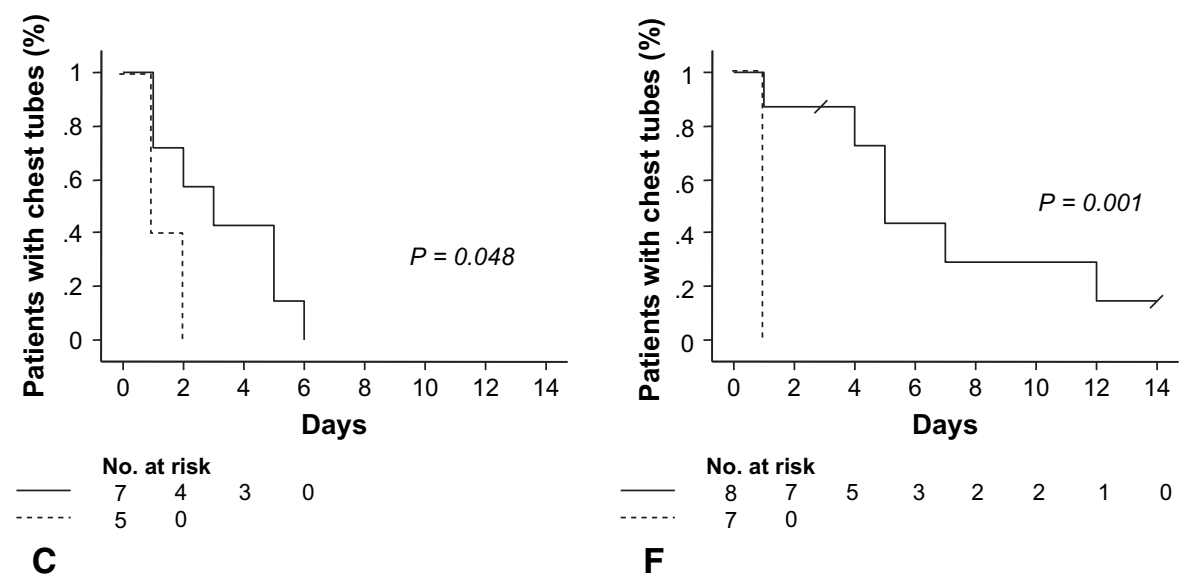

FIGURE 2. Percentage of patients with chest tubes in place on postoperative days 0 to 14 in the glue-alone (G) group (solid lines) and the mesh-and-glue (MG) group (dashed lines). The patients were divided into 6 subgroups based on the resection site (upper or lower lobe) and their emphysema index $(<1 \%$, $1 \%-10 \%$, and $>10 \%$ ): A, patients with an emphysema index of less than $1 \%$ who underwent lower lobe resection; B, patients with an emphysema index of $1 \%$ to $10 \%$ who underwent lower lobe resection; C, patients with an emphysema index of greater than $10 \%$ who underwent lower lobe resection; $\mathrm{D}$, patients with an emphysema index of less than $1 \%$ who underwent upper lobe resection; E, patients with an emphysema index of $1 \%$ to $10 \%$ who underwent upper lobe resection; and F, patients with an emphysema index of greater than $10 \%$ who underwent upper lobe resection. The duration of chest tube drainage was shorter in the MG group than in the G group in patients with any emphysema index who underwent upper lobe resection and in patients with an emphysema index of greater than $10 \%$ who underwent resection of another part of the lung. 
TABLE 2. Postoperative complications in the glue-alone and meshand-glue groups

\begin{tabular}{lcc}
\hline \multicolumn{1}{c}{ Complications } & G group $(\mathbf{n}=\mathbf{6 1})$ & MG group $(\mathbf{n}=\mathbf{6 1})$ \\
\hline Pulmonary complications & $4(7 \%)$ & $0(0 \%)^{*}$ \\
Pneumonia & 2 & 0 \\
Respiratory failure & 1 & 0 \\
Lobar atelectasis & 1 & 0 \\
Cardiac complications & $2(3 \%)$ & $2(3 \%)$ \\
Atrial fibrillation & 2 & 1 \\
Pulmonary embolism & 0 & 1 \\
Total & $6(10 \%)$ & $2(3 \%)$ \\
\hline$G$, Glue alone; $M G$, mesh and glue. $* P=.042$ versus the glue-alone group.
\end{tabular}

Upper lobe resection and compromised pulmonary function are known risk factors for prolonged air leakage after lung lobectomy, ${ }^{9-11}$ which is in accordance with the outcome of the $\mathrm{G}$ group patients: both the site of resection and the emphysema index were significantly associated with the duration of chest tube drainage according to stepwise Cox regression analysis. In contrast, air leaks were not frequently detected during the water-seal test in patients who had undergone upper lobe resection. These results support the idea that an air leak does not tend to develop during surgical intervention in such patients, but when it does, it is difficult to treat. Thus pleural tenting was done as an additional intervention to obliterate the residual intrapleural space in patients undergoing upper lobe resection, especially those with emphysema; however, this was found to be of little benefit to reduce postoperative air leaks. ${ }^{12}$ Fortunately, our method with biocompatible material, which does not require any additional intervention, proved extremely useful, especially for patients undergoing upper lobe resection or patients with severe pulmonary emphysema.

Pulmonary emphysema is often distributed heterogeneously, which can also be associated with prolonged air leak. To clarify this concern, we classified the craniocaudal distribution of emphysema in the $\mathrm{G}$ group patients with a certain type of pulmonary emphysema (emphysema index $>1 \%, \mathrm{n}=43$ ) based on a visual classification system proposed by the National Emphysema Treatment Trial Research Group. ${ }^{13}$ This group classified pulmonary emphysema as predominantly affecting the upper lobes, predominantly affecting the lower lobes, diffuse, or predominantly affecting the superior segments of the lower lobes; the latter 3 categories were grouped together as predominantly nonupper lobe emphysema. In our series the emphysema was classified as predominantly upper lobe emphysema in 27 $(63 \%)$ patients and as predominantly non-upper lobe emphysema in the remaining $16(37 \%)$ patients. The duration of chest tube drainage did not differ significantly between these 2 groups $(P=.7501, \log$-rank test). Sixteen $(59 \%)$ of the 27 patients with predominantly upper lobe emphysema underwent upper lobe resection, whereas $8(50 \%)$ of the 16 patients with predominantly non-upper lobe emphy- sema underwent lower lobe resection $\left(P=.5545, \chi^{2}\right.$ test $)$ : the duration of chest tube drainage was significantly longer in the 16 subgroup patients who had undergone upper lobe resection than in the 8 subgroup patients who had undergone lower lobe resection $(P=.0272, \log$-rank test). Thus we believe that the distribution of emphysema does not play a critical role in determining the duration of postoperative air leaks.

Despite its excellent clinical outcome, the bursting pressure after sealing with PGA mesh and glue, as evaluated by a continuous stepwise pressure increase test in an animal model, had decreased by POD 4, although the PGA mesh would not have been degraded by hydrolysis within this time. ${ }^{14}$ This reduced sealing potential is attributed to the biochemical properties of either PGA mesh or glue, and therefore we must validate the use of these bioabsorbable materials without suturing in patients whose wound healing is disturbed by underlying diseases, such as diabetes or liver cirrhosis.

The limitation of the present study is that the patients were not randomly assigned to the 2 study groups. To adjust comparison, we paired the nonrandomized groups by using the nearest available (1-to-1) matching method on the propensity score. We believe that the bias has been reduced enough to allow duration of chest tube drainage to be compared between the 2 groups. Furthermore, we believe that the outcome of pneumostasis in the MG group patients was excellent because the duration of chest tube drainage and the postoperative hospital stay of patients with an air leak detected intraoperatively were similar to those of patients without any air leak detected intraoperatively. Furthermore, neither upper lobe resection nor emphysema was significantly associated with the duration of air leaks in the MG group patients because we were able to remove the chest tubes on POD 1 in most (90\%) of the MS group patients. The additional possible limitation is that we analyzed the duration of chest tube drainage instead of the duration of air leak. However, we believe that the duration of chest tube drainage is a reliable surrogate for the duration of air leak because we removed the chest tubes the day after disappearance of the air leaks, regardless of pleural drainage.

Regardless of the promising efficacy of combining PGA mesh and glue, it has not yet been confirmed whether this modality reduces the incidence of postoperative pulmonary complication. However, the prevention of postoperative air leaks apparently reduces the duration of chest tube drainage, allowing patients to begin mobilizing earlier with less pain and effective ventilation. All these beneficial effects synergistically promote pulmonary rehabilitation. We previously reported that chest tube removal is the most critical factor to achieve pulmonary physiologic rehabilitation in patients undergoing major lung resection for cancer. ${ }^{15}$

The emphysema index has been used to quantify emphysema because the low-attenuation area depicted on CT 
scanning was found to correspond well with pathologically diagnosed emphysema. ${ }^{16}$ Nevertheless, the clinical usefulness of the emphysema index has not been emphasized because neither $\mathrm{FEV}_{1}$ nor the risk of death from chronic obstructive pulmonary disease or lung cancer correlated with this measurement. ${ }^{17-19}$ In contrast, we showed the usefulness of the emphysema index in predicting surgical outcome after major lung resection for cancer: the emphysema index, and not $\mathrm{FEV}_{1}$, was predictive of air leak duration, ${ }^{10}$ hypoxemia, ${ }^{20}$ and prognostic outcome,${ }^{21}$ especially in patients with a smoking history. We also found that the emphysema index together with $\mathrm{FEV}_{1}$ helps to identify patients with a high risk of postoperative cardiopulmonary complications $^{22}$ and prolonged rehabilitation. ${ }^{15}$ Thus we advocate measuring the emphysema index routinely in patients scheduled to undergo lung cancer surgery, especially considering that it does not subject the patient to invasive intervention.

In conclusion, the combination of PGA mesh and glue for pneumostasis can reduce the duration of chest tube drainage, the postoperative hospital stay, and the incidence of pulmonary complications after major lung resection for cancer. Patients undergoing upper lobe resection and patients with severe emphysema might be good candidates for this procedure. Thus a larger-scale study is warranted to confirm our results.

\section{References}

1. Serra-Mitjans M, Belda-Sanchis J, Rami-Porta R. Surgical sealant for preventing air leaks after pulmonary resections in patients with lung cancer. Cochrane Database Syst Rev. 2005;(3):CD003051.

2. Ueda K, Tanaka T, Jinbo M, Yagi T, Li T-S, Hamano K. Sutureless pneumostasis using polyglycolic acid mesh as artificial pleura during video-assisted major pulmonary resection. Ann Thorac Surg. 2007;84:1858-61.

3. Beckles MA, Spiro SG, Colice GL, Rudd RM. American College of Chest Physicians. The physiologic evaluation of patients with lung cancer being considered for resectional surgery. Chest. 2003;123:105S-14S.

4. Minato N, Shimokawa T, Katayama Y, Yamada N, Sakaguchi M, Itoh M. New application method of fibrin glue for more effective hemostasis in cardiovascular surgery: rub-and-spray method. Jpn J Thorac Cardiovasc Surg. 2004;52:361-6.

5. Nakajima S, Fukuda T, Hasue M, Sengoku Y, Haraoka J, Uchida T. New technique for application of fibrin sealant: rubbing method devised to prevent cerebro- spinal fluid leakage from dura mater sites repaired with expanded polytetrafluoroethylene surgical membranes. Neurosurgery. 2001;49:117-23.

6. Ueda K, Kaneda Y, Sakano H, Tanaka T, Li TS, Hamano K. Obstacles for shortening hospitalization after video-assisted pulmonary resection for lung cancer. Ann Thorac Surg. 2003;76:1816-20.

7. Lamers RJ, Thelissen GR, Kessels AG, Wouters EF, van Engelshoven JM. Chronic obstructive pulmonary disease: evaluation with spirometrically controlled CT lung densitometry. Radiology. 1994;193:109-13.

8. Kinsella M, Muller NL, Abboud RT, Morrison NJ, DyBuncio A. Quantitation of emphysema by computed tomography using a "density mask" program and correlation with pulmonary function tests. Chest. 1990;97:315-21.

9. Brunelli A, Monteverde M, Borri A, Salati M, Marasco RD, Fianchini A. Predictors of prolonged air leak after pulmonary lobectomy. Ann Thorac Surg. 2004;77: 1205-10.

10. Ueda K, Kaneda Y, Sudo M, Mitsutaka J, Li TS, Tanaka N, et al. Quantitative computed tomography versus spirometry in predicting air leak duration after major lung resection for cancer. Ann Thorac Surg. 2005;80:1853-8.

11. DeCamp MM, Blackstone EH, Naunheim KS, Krasna MJ, Wood DE, Meli YM, et al. Patient and surgical factors influencing air leak after lung volume reduction surgery: lessons learned from the National Emphysema Treatment Trial. Ann Thorac Surg. 2006;82:197-206.

12. Robinson LA, Preksto D. Pleural tenting during upper lobectomy decreases ches tube time and total hospitalization days. J Thorac Cardiovasc Surg. 1998;115: 319-26.

13. National Emphysema Treatment Trial Research Group. Patients at high risk of death after lung-volume-reduction surgery. N Engl J Med. 2001;345:1075-83.

14. Morikawa T, Katoh H. Improved techniques of applying fibrin glue in lung surgery. Eur Surg Res. 1999;31:180-6.

15. Ueda K, Sudoh M, Jinbo M, Li T-S, Suga K, Hamano K. Physiological rehabilitation after video-assisted lung lobectomy for cancer: A prospective study of measuring daily exercise and oxygenation capacity. Eur J Cardiothorac Surg 2006;30:533-7.

16. Madani A, Keyzer C, Gevenois PA. Quantitative computed tomography assessment of lung structure and function in pulmonary emphysema. Eur Respir J. 2001; 18:720-30

17. Gelb AF, Hogg JC, Muller NL, Schein MJ, Kuei J, Tashkin DP, et al. Contribution of emphysema and small airways in COPD. Chest. 1996;109:353-9.

18. Hole DJ, Watt GC, Davey-Smith G, Hart CL, Gillis CR, Hawthorne VM Impaired lung function and mortality risk in men and women: findings from the Renfrew and Paisley prospective population study. BMJ. 1996;313:711-5.

19. Kishi K, Gurney JW, Schroeder DR, Scanlon PD, Swensen SJ, Jett JR. The correlation of emphysema or airway obstruction with the risk of lung cancer: a matched case-controlled study. Eur Respir J. 2002;19:1093-8.

20. Ueda K, Kaneda Y, Sudou M, Jinbo M, Li TS, Suga K, et al. Prediction of hypoxemia after lung resection surgery. Interact Cardiovasc Thorac Surg. 2005;4: $85-9$.

21. Ueda K, Jinbo M, Li T-S, Yagi T, Suga K, Hamano K. Computed tomographydiagnosed emphysema, not airway obstruction, is associated with the prognostic outcome of early-stage lung cancer. Clin Cancer Res. 2006;12:6730-6.

22. Ueda K, Kaneda Y, Sudoh M, Jinbo M, Tanaka N, Suga K, et al. Role of quantitative $\mathrm{CT}$ in predicting hypoxemia and complications after lung lobectomy for cancer, with special reference to area of emphysema. Chest. 2005;128:3500-6. 